\title{
Posterior Column Dysfunction in Cervical Spondylotic Myelopathy
}

\author{
D.J. MacFadyen
}

\begin{abstract}
Five patients had cervical spondylotic myelopathy (CSM) with severe prorioceptive sensory loss in the upper and lower limbs. Despite an advanced age, each recovered considerable function following cervical laminectomy, in follow-up periods of 10 to 40 months. The role of newer methods of investigation including CT scanning, somatosensory evoked responses and nuclear magnetic resonance is discussed. The cause of the proprioceptive sensory loss in these patients cannot be definitively determined, but various ischemic and compressive lesions are discussed as possible pathogenetic mechanisms.
\end{abstract}

RÉSUMÉ: Cinq patients étaient porteurs d'une myélopathie spondylotique cervicale (MSC) avec une perte sévère de sensibilité proprioceptive dans les membres supérieurs. En dépit d'un âge avancé, chacun d'eux s'améliora considérablement sur le plan fonctionnel à la suite d'une laminectomie cervicale pendant une période d'observation de 10 à 40 mois. Des nouvelles méthodes d'investigation incluent la tomodensitométrie, les potentiels évoqués somato-sensoriels, la résonnance nucléaire magnétique comme suppléments diagnostiques à la myélopathie classique. La cause de la perte de la sensibilité proprioceptive chez ces patients ne peut être déterminée de façon définitive, mais des lésions variées ischémiques et compressives sont évoquées comme mécanismes pathogéniques possibles.

Can. J. Neurol. Sci. 1984; 11:365-370

Stookey (1928) was the first to report upon the surgical treatment of patients with cervical spondylotic myelopathy (CMS). He attributed their signs of spinal cord compression to an extradural chondroma and noted that the outcome following surgery was "not encouraging". Subsequent to studies by Schmorl and Andrae in which the "nature and structure" of the nucleus pulposus were elucidated, Stookey (1940) again reported on the subject and referred to herniation of the nucleus pulposus in the cervical region. Brain (1948) first discussed the importance of vascular factors in the production of CSM and distinguished between acute, usually traumatic cervical disc protrusion and chronic cervical disc disease and cord compression. In the same year Barnes (1948) emphasized cord injury resulting from hyperextension of a spondylotic cervical spine. The high frequency with which the radiological changes of cervical spondylosis (CS) appear in both males and females beyond the age of 50 and its increasing frequency with age has been well documented (Brain, 1963). The degree to which these radiological abnormalities correlate with signs of cervical cord or root dysfunction was addressed by Pallis et al. (1954).

In general, in CSM the signs secondary to myelopathy exceed those due to root compression, motor and reflex signs exceed sensory signs, analgesia exceeds anaesthesia, and both together exceed proprioceptive loss. Vibration sense loss appears ear- lier and exceeds that of joint position sense loss (Brain et al., 1952). Several reports describe individual patients in whom joint position sense loss was prominent (Brain et al., 1952; Mair et al., 1953; Stern and Rand, 1954; Clarke and Robinson, 1956). Valergakis (1976) emphasized CSM as a common cause of loss of position and vibratory senses. The following case reports are of patients with CSM in whom loss of joint position sense was particularly striking and in whom surgical treatment was successful in reversing the neurologic disability, at least in the short term.

Case 1. This 76 year old woman was admitted with a four month history of numbness of the hands and increasing ataxia of gait. She noted paresthesiae first in her left hand and then in her right hand, which progressed until she had difficulty with fine movements in the fingers. At the same time she experienced unsteadiness of gait which increased so that she eventually required a walker. There were no symptoms related to sphincters.

Examination demonstrated slight weakness of left hand grip and of hip flexors bilaterally with normal tone in all four limbs. There was ataxia of all four limbs. Pain and temperature sensation were normal in the legs and slightly impaired in the hands. Vibration sense (VS) was intact in the fingers but absent at the iliac crests. Joint position sense (JPS) was reduced in the fingers but was normal in the toes. Tendon reflexes were reduced in the upper limbs and were absent in the lower limbs. A Babinski reflex was present bilaterally.

Roentgenograms of the cervical spine showed marked degenerative changes (Figure 1). A myelogram was unsuccessful because of degenera-

From the Department of Clinical Neurological Sciences (Neurology) University of Saskatchewan, Saskatoon

Received December 13, 1983. Accepted in revised form May 1, 1984.

Reprint requests to: Dr. D.J. MacFadyen, Department of Clinical Neurosciences, University Hospital, Saskatoon, Saskatchewan, Canada S7N 0X0 
tive changes in the lumbar spine. A CT scan of the cervical spine revealed narrowing of the spinal canal below $\mathrm{C} 2$, degenerative changes in the vertebral bodies and articular facets from $\mathrm{C} 3$ to $\mathrm{C} 7$ and narrowing of the intervertebral foramina bilaterally from $\mathrm{C} 4$ to $\mathrm{C} 7$ (Figure 2).

A decompressive laminectomy from $\mathrm{C} 3$ to $\mathrm{C} 7$ was carried out. Within a few weeks the numbness in her right hand improved modestly as did her ataxia of gait. Twenty-seven months later she was living in her apartment but still had significant ataxia of gait and difficulty with fine movements in her hands. JPS was moderately reduced in the left fingers and in the toes bilaterally but was normal in the right fingers. VS was absent below the knees.

Case 2. This 80 year old woman had been very active until six weeks prior to admission when there was the onset of pain in the posterior neck and left shoulder. This was quickly followed by numbness of her left fingers, then both feet and then her right fingers. Upon extending her neck she noted tingling in her spine and in both hamstrings. The numbness spread proximally in all four limbs and increasing unsteadiness in gait ultimately allowed her to walk only with support. She had no sphincter symptoms.

There was very slight weakness in the left arm involving hand grip, finger abduction, wrist dorsiflexion and deltoid with slight bilateral weakness of hip flexion, hamstrings and ankle dorsiflexion and spasticity at the elbows and wrists. Slight left arm ataxia and ataxia of gait were demonstrated. VS was absent in the fingers and ankles and JPS was slightly decreased in the fingers and markedly decreased in the toes. The tendon reflexes were increased in the triceps and quadriceps femoris, decreased at the ankles, and a Babinski reflex was elicited bilaterally. Cervical spine X-rays showed minimal degenerative changes with slight forward displacement of $\mathrm{C} 4$ on $\mathrm{C} 5$ and $\mathrm{C} 5$ on $\mathrm{C} 6$. A myelogram revealed an almost complete block at the $\mathrm{C} 5$ level.

A decompressive laminectomy of C3-4-5-6 was carried out. Four months later she was walking with the aid of a cane. Strength in the legs

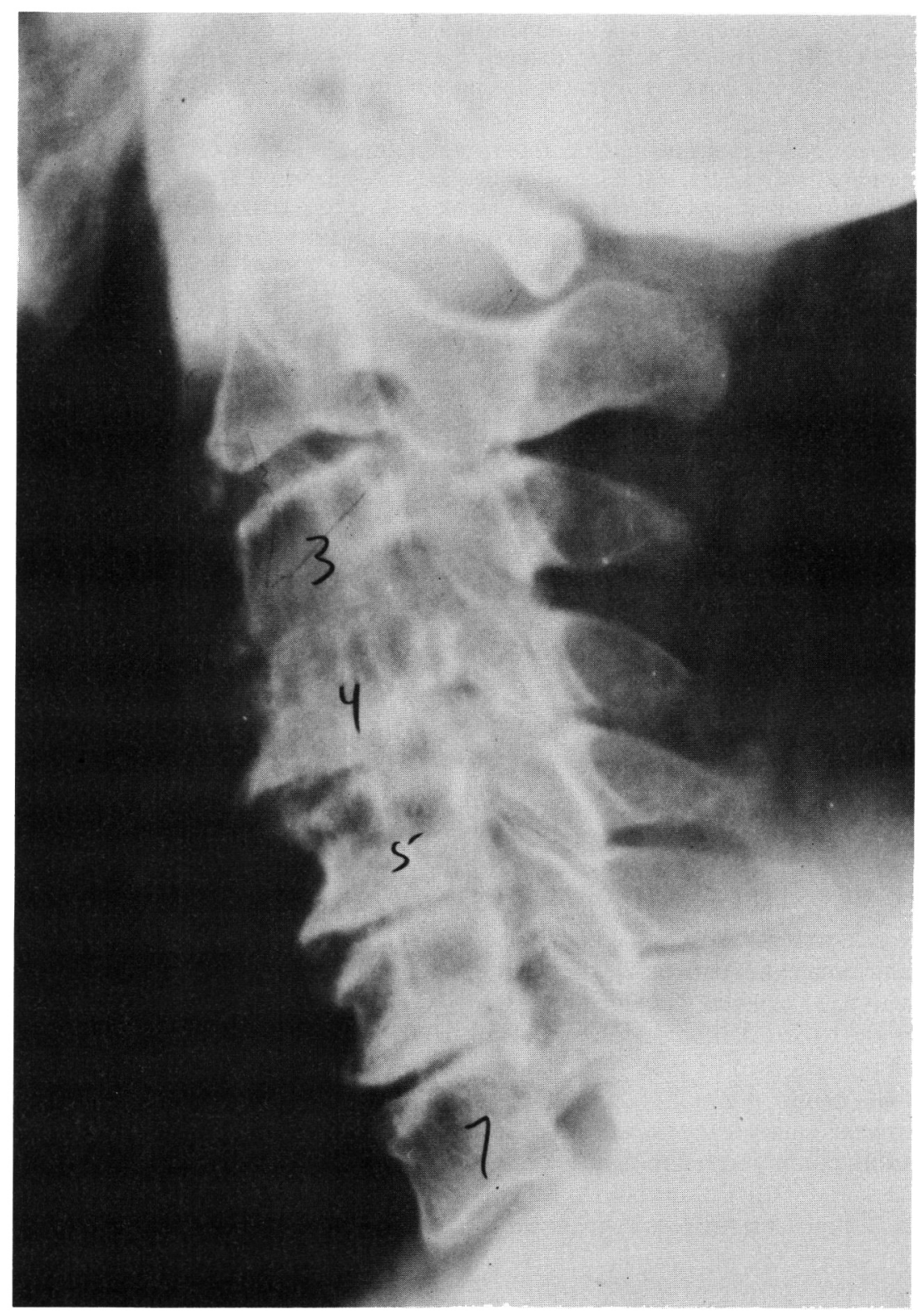

Figure I-Case 1: lateral view of cervical spine:illustrates marked disc degeneration at several levels with anterior and posterior osteophyte formation most markedly at C3-4 and C4.5 levels. 


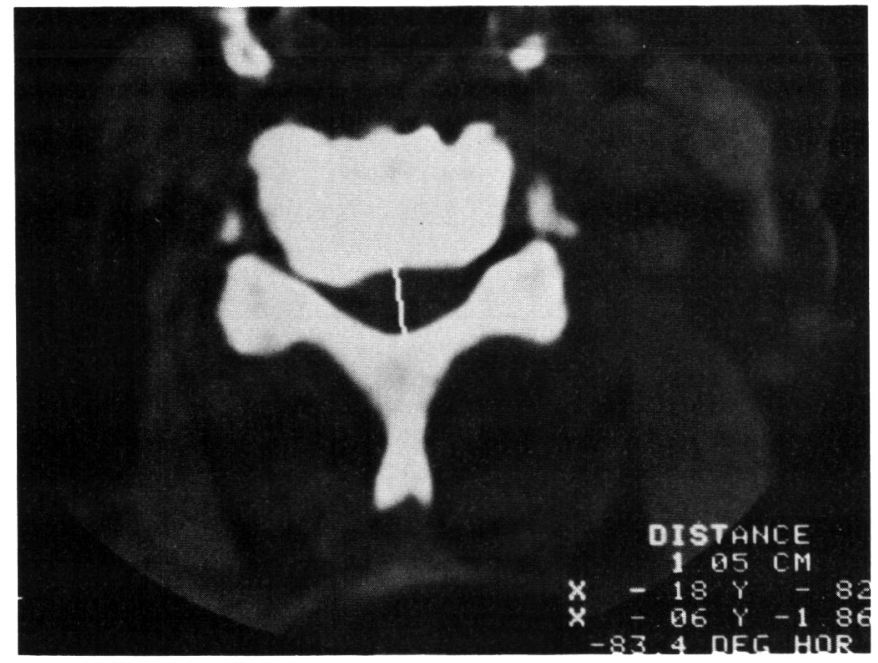

Figure 2-Case 1: CT scan of cervical spine at C6: illustrates spondylotic changes plus a narrowed AP canal diameter at $10.5 \mathrm{~mm}$.

and hands was good with normal position sense in the fingers. Superficial sensation was normal. Slight unsteadiness of gait secondary to spasticity in the legs and JPS loss in the toes remained. At $\mathbf{4 0}$ months she reported that her legs were "still troublesome" but she was physically very active and fully independent.

Case 3. This 82 year old female complained of pain in the right hip for two months. Two years prior to admisson she had had low back pain with radiation into both posterior thighs. Examination then revealed weakness of the paraspinal muscles, of knee flexion bilaterally and no (R) ankle plantar flexion. Tendon reflexes were absent in the legs but sensation was normal. For six months prior to this admission she had noted increasing difficulty with gait such that she required a walker upon admission. Use of her hands, especially in writing, had deteriorated.

Upon examination there was $4 / 5$ strength in all upper limb muscle groups except for the left triceps $(3+/ 5)$. Both arms were ataxic. There was 3-4/5 strength at the hips bilaterally with normal strength elsewhere except for $0 / 5$ in right ankle inversion. The upper limb tendon reflexes were hyperactive except for a diminished right triceps brachii reflex. Lower limb tendon reflexes were absent and there was a Babinski reflex bilaterally. Light touch was decreased only in the fingers bilaterally and stereognosis was markedly diminished in the hands bilaterally. Pain sensation was intact in the fingers but slightly decreased in the forearms and shins bilaterally. JPS was decreased in the upper and lower extremities. VS was not recorded. Cervical spine $\mathrm{x}$-rays showed narrowing of the C5-6 disc space with associated osteophyte formation but no narrowing of the spinal canal. A myelogram demonstrated an almost complete block at C5-6.

One month after a decompressive laminectomy from $\mathrm{C} 3$ to $\mathrm{C} 6$ the sensory loss to pain was unchanged but light touch and JPS were nearly normal in her extremities and she was able to identify five of six objects placed in either hand. She was able to transfer from wheelchair to bed and could walk 50 feet using a walker. Eight months following her laminectomy she was living in her son's home and walking with the aid of a walker.

Case 4. A 66 year old male had numbness and tingling of the right hand which had started in the thumb and index finger two years previously followed by increasing clumsiness in fine movements. Later similar numbness and tingling began in the left hand and in both arms, eventually spreading proximally to the elbows. Numbness and tingling in the feet extending proximally to the hips had been noted for three months with a perineal numbness present for six weeks. He had noted occasional frequency of voiding, some unsteadiness in walking but no limb weakness.

Examination revealed slight to moderate weakness of all muscle groups in the upper limbs with normal tone. Lower limb strength was normal with slight ataxia present in all four limbs. Light touch sensation was slightly diminished in both arms distal to the C5 dermatome. Pain and temperaiure sensation were slightly diminished in the left hand, the left foot and adjacent shin. VS was absent in all fingers and absent below the iliac crests. JPS was markedly decreased in the fingers and was moderately diminished in the toes. Except for a decreased left biceps brachii reflex the tendon reflexes were normal. There was no Babinski reflex. Cervical spine $X$-rays revealed a kyphosis in the mid-cervical area and extensive degenerative changes with marginal lipping and narrowed disc spaces in the mid cervical area. A myelogram showed an incomplete block at the C2-3 to C4-5 level with the cord displaced to the left and posteriorly. The AP diameter of the spinal canal at this level was $8 \mathrm{~mm}$. A cervical laminectomy from $\mathrm{C} 3$ to $\mathrm{C} 7$ was carried out, after which he noted rapid improvement in the sensation and dexterity in his hands. Four months later he had slight weakness in his left fingers, normal cutaneous sensation in the legs, normal reflexes and nearly normal gait. One year after his surgery his only symptom was slight numbness of his right little and ring fingers.

Case 5. A 72 year old woman was well until the age of 69 years when she fell striking her forehead on the floor. The following day she had tingling and numbness in the tips of all right fingers and less so in the left fingers. She had no limb weakness, difficulty with gait, or sphincter dysfunction. These symptoms were still present when seen three months later, but muscle bulk, power and tone were normal in all four limbs. The tendon reflexes were normal although slightly more brisk in the right limbs and there was a, left Babinski reflex. Light touch and VS were decreased in the right fingers but pain sensation and JPS were intact. X-rays of the cervical spine showed advanced disc space narrowing at C4-5 and C6-7 with associated anterior and posterior osteophytes. Over several months her symptoms disappeared. Three years later she again fell striking her face and shoulder. The following morning she noted pain in her neck with numbness and tingling of all fingers. The numbness increased over subsequent weeks until she no longer could write. There was increasing clumsiness of gait until she was confined to a wheelchair. Except for the deltoids there was weakness of all upper limb muscles, spasticity at the wrists, reduced fine movements in the fingers bilaterally and the right arm was slightly ataxic. Hip flexors, hamstrings and ankle dorsiflexors were weak bilaterally with spasticity at the knees. Touch, pain and temperature were decreased in all fingers. Touch was decreased in the lateral shins and pain distal to the knees. VS was absent or decreased in the fingers and absent below the right iliac crest and the left knee. JPS was decreased in the right fingers and in the toes bilaterally. Except for decreased biceps brachii and brachioradialis, the tendon reflexes were hyperactive, and Babinski reflexes were present bilaterally. A myelogram revealed generalized narrowing of the spinal canal in the lumbar and cervical regions, transverse ridges at several levels between $\mathrm{C} 3$ and $\mathrm{T} 1$ and a high grade obstruction at C3-4, C4-5 and C5-6.

The day following a cervical laminectomy from $\mathrm{C} 2$ to $\mathrm{C} 7$ there was obvious improvement in the fine movements and numbness of both hands. Ten months later she was able to walk for up to three hours and she had no sensory loss although her reflex changes remained.

\section{Discussion}

These five patients with CSM, summarized in Table 1, all underwent a cervical laminectomy. They are made up of four females and one male ranging in age from 66 to 82, with only one less than 70 years of age. In one case (5) trauma played a major role in the precipitation of symptoms. The duration of symptoms ranged from 6 weeks to 3 years and those with the longest duration generally improved most following surgery. Two patients (4 and 5) whose symptoms began in their upper limbs had made an excellent recovery upon follow-up at one year and ten months respectively. Two patients ( 2 and 3 ), four and eight months post-operatively, had improved significantly in spite of their advanced age and patient 2 has maintained this improvement for $\mathbf{4 0}$ months. Patient 1 had improved only modestly at two months but maintained this for 27 months.

The symptoms and signs with which CSM patients present are a measure of the degree to which the posterior, dorsolateral 


\begin{tabular}{|c|c|c|c|c|c|c|c|c|c|c|c|c|c|c|c|c|c|}
\hline \multirow{3}{*}{ Case } & \multirow{3}{*}{ Sex } & \multirow{3}{*}{ Age } & \multirow{3}{*}{$\begin{array}{c}\text { Duration } \\
\text { of } \\
\text { Symptoms }\end{array}$} & \multirow{3}{*}{$\begin{array}{c}\text { Main } \\
\text { Presenting } \\
\text { Symptoms(s) }\end{array}$} & \multirow{3}{*}{ Trauma } & \multirow{3}{*}{$\begin{array}{l}\text { Cervical } \\
\text { Nerve } \\
\text { Root } \\
\text { Signs }\end{array}$} & \multicolumn{8}{|c|}{ Physical findings } & \multirow{3}{*}{$\begin{array}{l}\text { Cer- } \\
\text { vical } \\
\text { lamin- } \\
\text { ectomy }\end{array}$} & \multirow{3}{*}{$\begin{array}{l}\text { Results } \\
\text { of } \\
\text { follow-up }\end{array}$} & \multirow{3}{*}{$\begin{array}{l}\text { Length } \\
\text { of } \\
\text { follow-up } \\
\text { months }\end{array}$} \\
\hline & & & & & & & \multicolumn{2}{|c|}{$\begin{array}{c}\text { Pyramidal } \\
\text { Tract }\end{array}$} & \multicolumn{2}{|c|}{$\begin{array}{l}\text { vs } \\
\text { loss }\end{array}$} & \multicolumn{2}{|c|}{$\begin{array}{l}\text { Jps } \\
\text { loss }\end{array}$} & \multicolumn{2}{|c|}{$\begin{array}{l}\text { Cutaneous } \\
\text { sensory } \\
\text { loss }\end{array}$} & & & \\
\hline & & & & & & & $\mathbf{U L}$ & $\mathbf{L L}$ & $\mathbf{U L}$ & $\mathbf{L L}$ & UL & $\mathbf{L L}$ & UL & $\mathbf{L L}$ & & & \\
\hline 2 & $\mathbf{F}$ & 80 & $6 \mathrm{wks}$ & $\begin{array}{l}\text { pain in neck, } \\
\text { numbness of } \\
\text { extremities, } \\
\text { ataxia of gait }\end{array}$ & 0 & 0 & + & + & ++ & ++ & + & +++ & 0 & 0 & + & $\begin{array}{l}\text { marked } \\
\text { improvement }\end{array}$ & 40 \\
\hline $4^{*}$ & $\mathbf{M}$ & 66 & $2 \mathrm{yrs}$ & $\begin{array}{l}\text { numbness of } \\
\text { hands, then } \\
\text { legs }\end{array}$ & 0 & o & $+?$ & o & ++ & +++ & +++ & ++ & + & + & + & $\begin{array}{l}\text { marked } \\
\text { improvement }\end{array}$ & 12 \\
\hline $5^{*}$ & $\mathbf{F}$ & 72 & $3 \mathrm{yrs}$ & $\begin{array}{l}\text { numbness of } \\
\text { fingers, ataxia } \\
\text { of gait }\end{array}$ & + & $+1-$ & + & ++ & ++ & +++ & ++ & ++ & ++ & ++ & + & $\begin{array}{l}\text { marked } \\
\text { improvement }\end{array}$ & 10 \\
\hline
\end{tabular}

\footnotetext{
$+=$ slight involvement/loss

$++=$ moderate involvement/loss

$+++=$ marked involvement/loss

* from the Department of Medicine (neurology) and Surgery (neurosurgery), Vancouver General Hospital
}

and ventrolateral columns, the ventral horns and the cervical nerve roots are involved. In each patient more than one of these structures is usually involved. Crandall and Batzdorff (1966) describe five categories of clinical presentation in a series of 62 patients: (1) transverse lesion syndrome (29/62) with equal involvement of corticospinal, spinothalamic and posterior columns and where the duration of the disease is longest in this group; (2) motor system syndrome (12/62): corticospinal or anterior horn cell involvement with minimal sensory symptoms and signs; (3) central core syndrome (8/62): motor and sensory involvement affecting upper limbs more than lower and all with "useless" hands; (4) Brown-Sequard syndrome (8/62) and (5) brachialgia and cord syndrome (5/62): upper limb pain and some long tract involvement. Even hemiparesis has been described in CSM (Wallach et al., 1976). Our five patients most closely resemble the central core syndrome.

The natural history of the disease is generally one of slow, prolonged deterioration. Acute exacerbations may be superimposed especially where there is a hyperextension neck injury or rheumatoid arthritis. Periods in which little or no progression occurs are common and this complicates the assessment of the effectiveness of conservative or surgical therapy. A significant number of patients improve or at least stop deteriorating following surgery only to subsequently resume their progressive course. The duration of symptoms prior to diagnosis varies considerably and is determined by a number of factors one of which may be that CSM patients with a normal "developmental" spinal canal AP diameter tend to have a longer prediagnosic stage than do patients with a narrowed spinal canal (Edwards and LaRocca, 1983).
The pathology and vascularity of the cord in CSM have been described in detail by several authors (Brain et al., 1952; Mair and Druckman, 1953; Wilkinson, 1960; Breig et al., 1966; Ogino et al., 1983) whereas the skeletal pathology has been described in other reviews (Payne and Spillane, 1957; ten Have and Euldernik, 1980). With the exception of the status of the extrinsic blood supply to the cervical cord there is a marked similarity in the histologic findings in these various reviews of the pathology in CSM regardless of patient age, duration of symptoms, severity of spondylotic changes, developmental canal AP diameter and with or without trauma. These findings include flattening of the cervical cord with indentation corresponding to underlying spondylotic ridges (these may be fixation artefacts), consistent demyelination of the corticospinal tracts, frequent demyelination of the anteromedial posterior columns and of the anterolateral columns. The anterior columns are consistently spared in spite of their proximity to the spondylotic protrusions. Neuronophagia and neuron cell loss is commonly found in the ventral horns and occasionally in the dorsal horns. At the least these findings are accompanied by gliosis and, in severe cases, by frank cavitation or tissue destruction. Dorsal root fibers may be demyelinated with axonal loss and the ventral root fibers are decreased or atrophic secondary to either loss of ventral horn neurons or nerve root compresson. The degree to which the arterial supply of the cervical cord is affected seems to vary from a report of "thick collagenous walls in small vessels" (Nair and Druckman, 1953) to "no atherosclerotic change in the extrinsic and intrinsic vasculature of the spinal cord and its coverings" (Ogino et al., 1983). 
This uniformity in the pathology of CSM is possibly due to the limited way in which the cervical cord reacts histologically to varied pathological processes. There may be some pathophysiological "final common pathway," one element of which is the intrinsic vascularity of the cord. Turnbull et al. (1966) addressed this possibility studying first the microangiographic blood supply of the cervical cord in unselected cadavers and then in CS without myelopathy (Breig et al., 1966). They suggested that the "number and location of the radicular arteries may be highly relevant" and that the consistent involvement of the lateral columns in CSM is the result of lengthening and narrowing of the horizontally oriented penetrating pial arteries caused by the widening of the cord secondary to compression by the spondylotic ridges, whereas the sparing of the outer half of the posterior columns is the result of lack of compression of their arterial supply which is oriented in the same direction as the compressing force, i.e. sagittally. However, this explanation does not explain the consistent sparing of the anterior columns. Mair and Druckman (1953) suggested that those parts of the cord involved represent the distal distribution of the anterior spinal artery, and this has subsequently been described as a "watershed" lesion (Taylor, 1964; Ogino et al., 1983). Gooding et al. (1975), studied the effects of a combination of progressive cervical cord compression by means of a screw, selective ligation of arteries supplying the cord and hypotension secondary to bleeding on the canine spinal cord. They concluded that local ischemia of the cervical cord caused by local deformation when superimposed on a regional reduction in spinal cord blood flow accounts for CSM both in the experimental setting and in humans. On the other hand Nurick (1972), points out that patients with CSM and general vascular disease are not more severely affected or worse in prognosis than those without vascular disease and that because patients with cervical root symptoms seldom develop CSM reduced radicular arterial supply to the cord secondary to root sleeve fibrosis is an unlikely cause.

Whatever the role of the blood supply to the cord in the production of CSM there can be no doubt that a nearly universal finding is a decreased AP diameter of the cervical spinal canal secondary to degenerative disc disease $+/$ - developmental narrowing of the canal. Related factors which may play a role include infolding and/or hypertrophy of the ligamentum flavum (Stoltmann and Blackwood, 1964), ossification of the posterior longitudinal ligament (Murakami et al., 1978) and subluxation or increased mobility of one vertebral body upon another with, in the case of marked mobility, kyphosis of the cervical spine upon flexion. Barnes and Saunders (1983) found that those patients who did deteriorate following a period of conservative therapy had a significantly higher degree of cervical mobility when compared to those whose disability had remained static. Adams and Logue (1971) concluded that traction of the spinal cord against posterior osteophytes is important in the production of CSM when there is an excessive degree of kyphosis of the neck during flexion and when the dural sac and cord are under tension.

We have selected our five patients because of their striking sensory findings and especially loss of joint position sense, but we cannot explain the cause of this sensory loss. Adam and Logue (1971) suggest that pain and temperature impairment in the lower limbs tends to occur when there is either an appreciable degree of kyphosis or subluxation in flexion. They and other authors also point out that sensory loss in the upper limbs is difficult to specifically attribute to either nerve root or cord lesions. It is only in the case of pain and temperature sensation that the primacy of a specific tract within the spinal cord is well established and even here it is clear that, following spinothalamic tract section, alternate pathways exist. Wall (1970) states that no sensory defect has been shown to follow isolated dorsal column lesions but it is observed when combined with lesions of other tracts. Subsequently, Wall and Noordenbos (1977) studied three patients with surgically confirmed lesions of the dorsal columns and after testing sensation in a variety of ways concluded that there was no loss of the classical primary modalities of sensation but loss of an ability to carry out tasks where one must simultaneously analyze spatial and temporal characteristics of the stimulus. A review of their methods of testing for JPS suggests that it was probably markedly or completely lost especially since one can accurately detect 0.5 degrees of movement at the hip even after total hip replacement surgery (Karenjia and Ferguson, 1983) and 4.4 degrees of movement at the great toe (Browne et al., 1954). In the case of vibration sense, Calne and Pallis (1966) propose that it represents a temporal modulation of tactile sense, that it is not a specific "modality," that the relevant impulses ascend in both the dorsal and lateral columns, and that the physiological implications of "posterior column signs" are redundant as they are based upon inadequate clinicopathological correlations.

The diagnostic criteria for CSM consisting of the history, physical findings and the radiological changes in the cervical spine and upon myelography have remained constant for many years. The degree to which newer investigative techiques will contribute to clarification of diagnosis, prognosis and treatment selection is not yet clear. It would seem that somatosensory evoked responses recorded from the brachial plexus, the cervical spine and the cortex are normal in those patients with only cervical spondylosis, may be abnormal in those patients with CSM where the dorsal column/medial lemniscal system is involved and have the best correlation with other diagnostic criteria in cervical radiculopathy especially in association with loss of JPS, vibration sense or impaired two-point discrimination (Ganes, 1980; Eisen et al., 1983). The same modality-SSER correlation occurs in multiple sclerosis (Namerow, 1968). The role of CT scanning in CSM is also evolving and its utility undoubtedly depends upon whether the scanner used can visualize the cervical cord. Although CT scanning without contrast can be of value in detecting intervertebral canal abnormalities, soft disc protrusions and spondylosis, it is technically demanding and not universally applicable. The addition of water-soluble myelography adds to its specificity and usefulness in the investigation of CSM but its merits relative to conventional myelography are still evolving (Scotti et al., 1983; Nakagawa, 1983). Magnetic resonance is even more unknown as to its value although its ability to image the entire length of the spinal cord, but with limited visualization of skeletal structure, is impressive.

The treatment of CSM consists of immobilization of the cervical spine by means of a collar or surgical intervention in the form of an extensive laminectomy or an anterior discectomy and fusion (Cloward procedure). Although immobilization by means of a collar has long been recognized as valuable and its utility, especially in the presence of an abnormally mobile spine has been emphasized by Barnes and Saunders (1983), lack of compliance which is probably proportional to the degree of 
immobilization achieved is a major disadvantage. The relative merits of a laminectomy as compared to the Cloward procedure are difficult to determine and are probably unknown except in specific circumstances. Where pathology at a specific disc space is responsible for cervical cord compression and radiculopathy the Cloward procedure probably excels whereas where multiple disc levels are involved and excessive cervical spine mobility is not a problem laminectomy is preferable. Typical of the results obtained by laminectomy are those reported by Nurick (1972) where, in 474 patients with CSM, 56\% improved, $25 \%$ were unchanged and $19 \%$ worsened.

Of particular note in the five patients I have described is the encouraging result of surgery at least in the short term and in spite of their advanced age. Indeed it may be that patients with CSM in whom proprioceptive sensory loss is a major feature constitute a group in whom cervical cord decompression produces a particularly favourable result. Why these patients should so benefit or why they have this major proprioceptive sensory loss is not clear. It seems unlikely that the conventional methods of investigation of patients with CSM will further clarify the etiology of CSM or provide significant further improvement in patient selection for particular types of treatment. The degree to which newer methods of investigation such as CT scanning, evoked response studies and MRI will increase our knowledge of the patho-physiology of CSM or improve our treatment selection remains to be determined.

\section{REFERENCES}

Adams CBT, Logue V (1971) Studies in cervical spondylotic myelopathy. Brain, 94: 557-594.

Barnes MP, Saunders M (1983) The effect of cervical mobility on the natural history of cervical spondylotic myelopathy. Can. J. Neurol. Sci., 10: 130.

Barnes R (1948) Paraplegia in cervical spine injuries. J. Bone \& Joint Surg., 30: 234-248.

Brain WR (1948) Discussion on rupture of the intervertebral disc in the cervical region. Proc. Roy. Soc. Med., 41: 509-511.

Brain WR, Northfield D, Wilkinson M (1952) The neurological manifestations of cervical spondylosis. Brain, 75: 187-225.

Brain, Lord (1963) Some unsolved problems of cervical spondylosis. Brit. Med. J., i: 771-777.

Breig A, Turnbull IM, Hassler O (1966) Effects of mechanical stresses on the spinal cord in cervical spondylosis. A study on fresh cadaver material. J. Neurosurg., 25: 45-65.

Browne K, Lee J, Ring PA (1954) The sensation of passive movement at the metatarsal-phalangeal joint in the great toe in man. J. Physiol. (London), 126: 448-458.

Calne DB, Pallis CA (1966) Vibration sense: a critical review. Brain, 89: 723-746.

Clarke E, Robinson PK (1956) Cervical myelopathy: a complication of cervical spondylosis. Brain, 79: 483-510.

Crandall PH, Batzdorff V (1966) Cervical spondylotic myelopathy. J. Neurosurg., 25: 57-66.

Edwards WC, LaRocca H (1983) The developmental sagittal diameter of the cervical spinal canal with cervical spondylosis. Spine, 8: 20-27.
Eisen A, Hoirch M, Moll A (1983) Evaluation of radiculopathies by segmental stimulation and somatosensory evoked potentials. Can. J. Neurol. Sci., 10: 178-182.

Ganes T (1980) Somatosensory conduction times and peripheral, cervical and cortical evoked potentials in patients with cervical spondylosis. J. Neurol., Neurosurg. \& Psychiat., 43: 683-689.

Gooding MR, Wilson CB, Hoff JT (1975) Experimental cervical myelopathy: effects of ischemia and compression of the canine cervical spinal cord. J. Neurosurg., 43: 9-17.

Karanjia PN, Ferguson JH (1983) Passive joint position sense after total hip replacement surgery. Ann. Neurol., 13: 654-657.

Mair WGP, Druckman R (1953) The pathology of spinal cord lesions and their relation to the clinical features in protrusion of cervical intervertebral discs. Brain. 76: 70-91.

Murakami N et al. (1978) Cervical myelopathy due to ossification of the posterior longitudinal ligament. Arch. Neurol., 35: 33-36.

Nakagawa H, Okumura T, Sugiyama T, Iwata K (1983) Discrepancy between metrizamide $\mathrm{CT}$ and myelography in diagnosis of cervical disc protrusion. Am. J. Neuroradiology, 4: 604-606.

Namerow NS (1968) Somatosensory evoked responses in multiple sclerosis patients with varying sensory loss. Neurology, 18: $1197-1204$

Nurick S (1972) The pathogenesis of the spinal cord disorder associated with cervical spondylosis. Brain, 95: 87-100.

Nurick S (1972) The natural history and the results of surgical treatment of the spinal cord disorder associated with cervical spondylosis. Brain, 95: 101-108.

Ogino $\mathrm{H}$ et al. (1983) Canal diameter, anteroposterior compression ratio, and spondylotic myelopathy of the cervical spine. Spine, 8: 1-15.

Pallis C, Jones AM, Spillane JD (1954) Cervical spondylosis: incidence and implications. Brain, 77: 274-289.

Payne EE, Spillane JD (1957) The cervical spine: an anatomicopathological study of 70 specimens with particular reference to the problem of cervical spondylosis. Brain, 80: 571-596.

Scotti G, Scialfa G, Pieralli S, Tonon C, Boccardi E (1983) Myelopathy and radiculopathy due to cervical spondylosis: myelographic CT correlations. Am. J. Neuroradiology, 4: 601-603.

Stern WE, Rand RW (1954) Spinal cord dysfunction from cervical intervertebral disk disease. Neurology, 4: 883-893.

Stoltmann HF, Blackwood W (1964) The role of the ligamenta flava in the pathogenesis of myelopathy in cervical spondylosis. Brain, 87: 45-50.

Stookey B (1928) Compression of the spinal cord due to ventral extradural cervical chondroma. Arch. Neurol. Psychiat., 20: 275-291.

Stookey B (1940) Compression of the spinal cord and nerve roots by herniation of the nucleus pulposus in the cervical region. Arch. Surg., 40: 417-432.

Taylor AR (1964) Vascular factors in the myelopathy associated with cervical spondylosis. Neurology 14: 62-68.

ten Have HAMJ, Euldernik F (1980) Degenerative changes in the cervical spine and their relationship to its mobility. J. Pathol., 132: 133-159.

Turnbull IM, Breig A, Hassler O (1966) Blood supply of the cervical spinal cord in man: a microangiographic cadaver study. J. Neurosurg., 24: 951-965.

Valergakis FEG (1976) Cervical spondylosis: most common cause of position and vibratory sense loss. Geriatrics, 31: 51-56.

Wall PD (1970) The sensory and motor role of impulses travelling in the dorsal columns towards cerebral cortex. Brain, 93: 505-524.

Wall PD, Noordenbox W (1977) Sensory functions which remain in man after complete transection of dorsal columns. Brain, 100: 641-653.

Wallack EM et al. (1976) Hemiparesis in cervical spondylosis. J.A.M.A. 236: $2524-2525$.

Wilkinson M (1960) The morbid anatomy of cervical spondylosis and myelopathy. Brain, 83: 589-617. 